\title{
Aberration Corrected STEM-EELS: Applications to Magnetic Materials
}

M. Varela***, J. Gazquez***, F. Y. Bruno**, J. Garcia-Barriocanal**, M. A. Torija***, M. Sharma***, W. Luo****, M. P. Oxley****, M. Prange****, S. T. Pantelides****, C. Leighton***, C. Leon**, J. Santamaria** and S. J. Pennycook*

* Oak Ridge National Laboratory, Oak Ridge, TN 37831

** Universidad Complutense de Madrid. Madrid 28040, Spain

*** University of Minnesota, Minneapolis, MN 55455

**** Vanderbilt University, Nashville, TN 37235

The macroscopic properties of magnetic materials, thin films and nanosystems are intimately related to their atomic structure and the presence of defects, interfaces etc. Electron energy loss spectroscopy (EELS) in the aberration corrected scanning transmission electron microscope (STEM) allows simultaneous exploration of structure, chemistry and electronic properties in real space with atomic resolution. Furthermore, derived techniques such as electron magnetic chiral dichroism allow studies of magnetization in real space [1]. These techniques combine to provide a unique tool to understand systems such as the $\mathrm{La}_{0.7} \mathrm{Sr}_{0.3} \mathrm{MnO}_{3} / \mathrm{SrTiO}_{3}$ (LSMO/STO) ferromagnetic/insulating superlattices (Fig. 1) where unexpected behaviors such as a magnetic moment on Ti atoms have been measured. EELS images show that charge transfer takes place due to the presence of an extra interfacial $\mathrm{La}_{0.7} \mathrm{Sr}_{03}-\mathrm{O}$ plane [2]. In this talk we will review applications of STEM-EELS to such systems and show how interface charge transfer explains the observed ferromagnetism.

Other examples to be described include the sensitivity of the EELS fine structure to the spin state of atoms [3]. For example, $\mathrm{Co}$ ions in $\mathrm{A}_{1-\mathrm{x}} \mathrm{B}_{\mathrm{x}} \mathrm{CoO}_{3}$ perovskites (where $\mathrm{A}$ and $\mathrm{B}$ are divalent/trivalent elements) exhibit a competition between the crystal field splitting and Hund's-rule exchange energy in the $3 d$ states, which determines the spin state of the individual Co ions. These materials provide an ideal test bed to explore how environmental effects, such as $\mathrm{O}$ vacancies or impurities, affect the resulting $\mathrm{Co}$ spin state. The ordering of $\mathrm{O}$ vacancies along with the presence of epitaxial strain in these compounds can stabilize a superlattice in the Co spin state which can be measured by EELS [4]. Fig. 2 shows a simulated EELS spectrum from two inequivalent Co-O planes in a $\mathrm{La}_{0.5} \mathrm{Sr}_{0.5} \mathrm{CoO}_{3-\delta}$ (LSCO) thin film where strain has been partially released. A brownmillerite-like structure is observed due to the ordering of $\mathrm{O}$ vacancies. Important changes related to the $\mathrm{O}$ deficiency are observed around the onset of the edge, at the pre-peak feature. The calculated prepeak intensity is lower in the plane where the $\mathrm{O}$ vacancies are located. Calculations predict that the total 3d band occupations for Co atoms in both planes are the same, but the projected densities of states (PDOS) on those inequivalent Co positions show that Co atoms in the $\mathrm{O}$ vacancy plane are in a high spin state while Co atoms in the fully oxygenated plane are in a low spin state. Atomic resolution spectrum images indeed confirm that there is a superlattice in the pre-peak intensity (Fig 2 ), which is lower on the Co-O planes with oxygen vacancies. This example shows how column-bycolumn spectroscopy offers the possibility of atomic resolution mapping of spin states [4].

References

[1] P. Schattschneider et al., Nature 441 (2006) 486.

[2] J. Garcia-Barriocanal et al., Nature Comm.1 (2010) 82.

[3] R. Klie et al., Phys. Rev. Lett. 99 (2007) 047203. 
[4] J. Gazquez et al., Nanoletters ASAP (2011).

[5] This work was supported by the Office of Science, Materials Sciences and Engineering Division of the US Department of Energy and by the McMinn Endowment at Vanderbilt University. J. G. acknowledges financial support from the European Research Council Starting Investigator Award. Work at UMN was supported by NSF and DoE (neutron characterization).
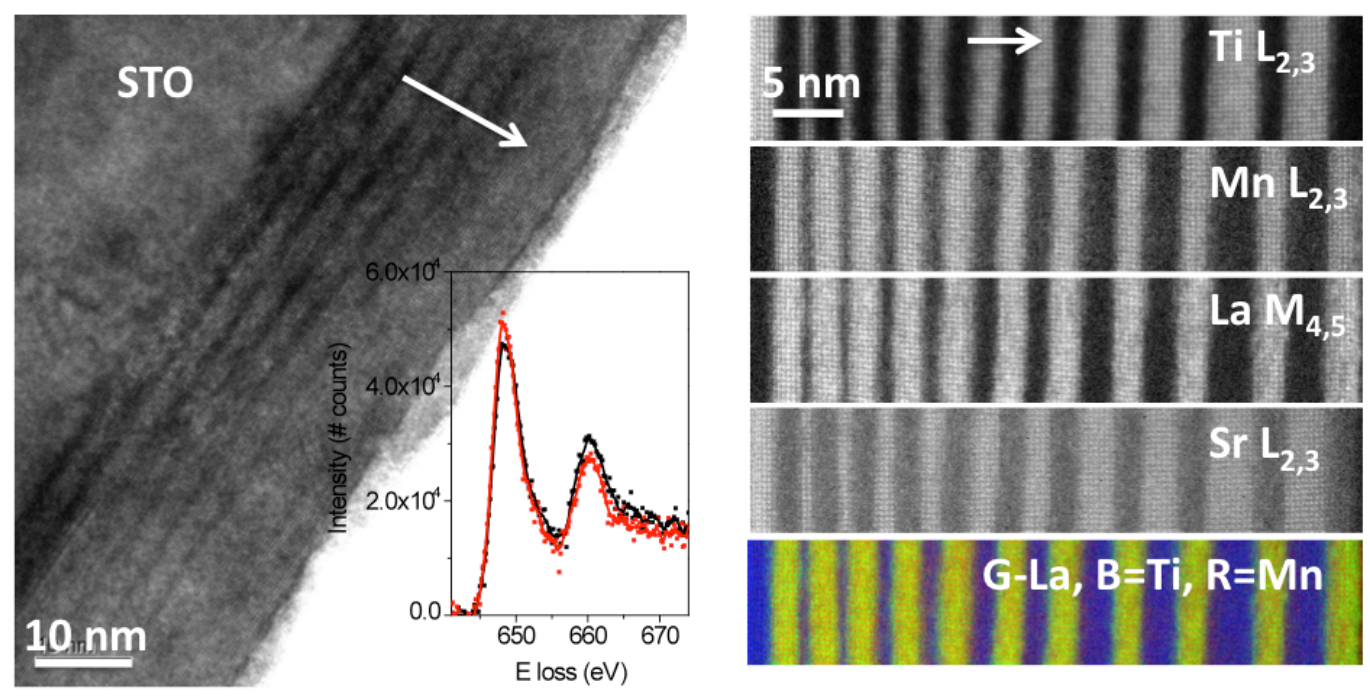

FIG. 1. (Left) TEM image of a LSMO/STO superlattice, acquired in a JEOL3000 microscope. Inset: $\mathrm{Mn} \mathrm{L}_{2,3}$ edges acquired at $97 \mathrm{~K}$ from two conjugate positions in the electron diffraction pattern, showing a clear dichroic signal. (Right) EELS elemental images of another $\mathrm{LSMO}_{6 \text { u.c. }} / \mathrm{STO}_{\text {n u.c. }}$. superlattice with changing n, obtained from a 2D spectrum image in the Nion UltraSTEM 100 after applying principal component analysis. The different elemental lattices can be clearly resolved allowing detailed interface structure studies. The growth direction is marked with a white arrow.
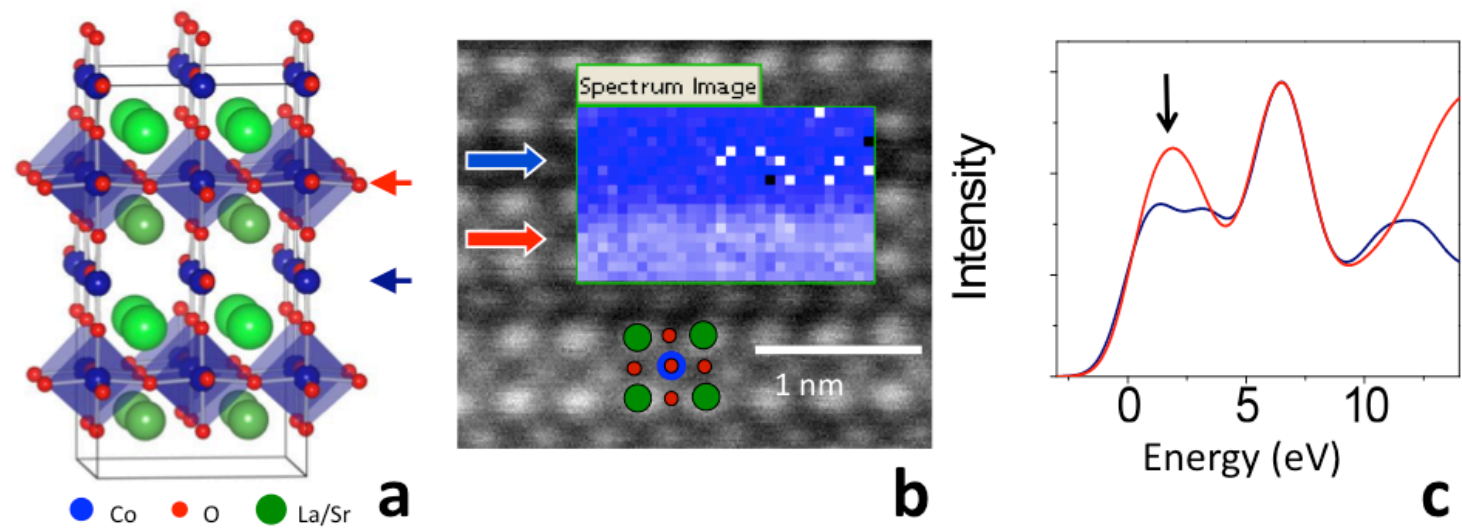

FIG. 2. (a) Brownmillerite derived structure used to simulate the $\mathrm{La}_{0.5} \mathrm{Sr}_{0.5} \mathrm{CoO}_{2.25}$ compound. A red (blue) arrow shows the fully oxygenated (O deficient) Co-O plane. The same color code is used for the rest of the figure. (b) Z-contrast image from the LSCO film, showing the alternate Co-O planes with (inset) a normalized pre-peak intensity map. Data from the VG Microscopes HB501UX, operated at $100 \mathrm{kV}$ and equipped with a Nion aberration corrector and a Gatan Enfina spectrometer.

(c) Simulated $\mathrm{O} K$ edges for the $\mathrm{La}_{0.5} \mathrm{Sr}_{0.5} \mathrm{CoO}_{2.25}$ compound. Adapted from [4]. 